\section{(2) OPEN ACCESS}

\title{
Native joint infections in Iceland 2003-2017: an increase in postarthroscopic infections
}

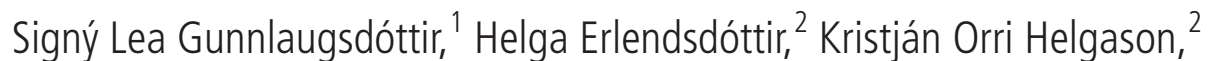
Árni Jón Geirsson, ${ }^{3}$ Valtýr Thors, ${ }^{4}$ Sigurður Guðmundsson, ${ }^{5,6}$ Magnús Gottfreðsson (iD 5,6

\begin{abstract}
Handling editor Josef $S$ Smolen

- Additional supplemental material is published online only. To view, please visit the journal online (http://dx.doi. org/10.1136/annrheumdis2021-220820).
\end{abstract}

${ }^{1}$ Medicine, Landspítali University Hospital, Reykjavík, Iceland ${ }^{2}$ Clinical Microbiology, Landspítali University Hospital, Reykjavík, Iceland

${ }^{3}$ Rheumatology, Landspitali, Reykjavik, Iceland

${ }^{4}$ Paediatrics, Landspitali, Reykjavik, Iceland

${ }^{5}$ Infectious Diseases, Landspitali, Reykjavik, Iceland

${ }^{6}$ Medicine, University of Iceland School of Health Sciences,

Reykjavik, Iceland

\section{Correspondence to}

Professor Magnús Gottfreðsson, Infectious diseases, Landspitali, Reykjavik, Iceland;

magnusgo@landspitali.is

Received 20 May 2021 Accepted 27 July 2021 Published Online First 17 September 2021

\section{Check for updates}

(C) Author(s) (or their employer(s)) 2022. Re-use permitted under CC BY-NC. No commercial re-use. See rights and permissions. Published by BMJ.

To cite: Gunnlaugsdóttir SL, Erlendsdóttir $\mathrm{H}$, Helgason $\mathrm{KO}$, et al. Ann Rheum Dis

2022:81:132-139.

\section{ABSTRACT}

Objectives Nationwide study on the epidemiology, clinical characteristics and outcomes among patients with native joint infection (NJ) in Iceland, 2003-2017. Methods All positive synovial fluid culture results in Iceland were identified and medical records reviewed. Results A total of $299 \mathrm{NJI}$ (40 children and 259 adults) were diagnosed in Iceland in 2003-2017, with a stable incidence of 6.3 cases/100 000/year, but marked gender difference among adults (33\% women vs $67 \%$ men, $p<0.001)$. The knee joint was most commonly affected, and Staphylococcus aureus was the most common isolate in both adults and children, followed by various streptococcal species in adults and Kingella kingae in children. $\mathrm{NJI}$ was iatrogenic in $34 \%$ of adults (88/259) but comprised $45 \%$ among $18-65$ years and a stable incidence. Incidence of infections following arthroscopic procedures in adults increased significantly compared with the previous decade (9/100 000/year in 1990-2002 vs 25/100 000/year in 2003-2017, $p<0.01$ ) with no significant increase seen in risk per procedure. The proportion of postarthroscopic NJI was $0.17 \%$ overall but $0.24 \%$ for knee arthroscopy. Patients with postarthroscopic infection were more likely to undergo subsequent arthroplasty when compared with other patients with NJI $(p=0.008)$.

Conclusions The incidence of $\mathrm{NJ}$ in Iceland has remained stable. The proportion of iatrogenic infections is high, especially among young adults, with an increase seen in postarthroscopic infections when compared with the previous decade. Although rare, NJI following arthroscopy can be a devastating complication, with significant morbidity and these results, therefore, emphasise the need for firm indications when arthroscopic treatment is considered.

\section{INTRODUCTION}

Bacterial septic arthritis (SA) is an uncommon yet serious infection which can lead to rapid destruction of the joint. These infections often require a lengthy hospital stay and prolonged treatment with intravenous antibiotics, resulting in high patient and healthcare burden. Delayed or inadequate treatment can result in permanent loss of joint function as well as life-threatening septicaemia. Even with treatment, overall mortality rates related to SA can be significant in adults, ranging from $3 \%$ to $23 \%$ with 30 -day mortality rates of $2 \%-10 \%{ }^{1-8}$ and a poor functional outcome in $24 \%$ of cases. ${ }^{9}$

\section{Key messages}

What is already known about this subject?

- There are few studies on native joint septic arthritis (native joint infection, NJI), which address the impact of iatrogenic infections.

What does this study add?

- This study confirms a high proportion of iatrogenic NJI in Iceland, with an increase seen in postarthroscopic infections when compared with the previous decade.

- Our nationwide analysis estimated the frequency of postarthroscopic NJI to be $0.17 \%$ rising to $0.24 \%$ for the knee joint.

How might this impact on clinical practice or
future developments?
Although rare, there are serious complications
associated with arthroscopy, including infection
which can cause considerable morbidity and
potential sequelae.
Appreciation of these risks and establishment
of firm indications for arthroscopic procedures
is essential.

The incidence of SA is increasing according to some studies. ${ }^{51011}$ This can potentially be explained by an ageing population, increased use of immunosuppressive drugs and an increase in invasive diagnostic and therapeutic joint procedures such as joint injections, arthroscopies and open joint surgery. Arthroscopic procedures have increased steadily in the last two decades, largely supplanting open joint surgery (other than joint replacement surgery). The most common indications for arthroscopic procedures in adults are degenerative or traumatic meniscal tears and arthroscopic partial meniscectomy (APM) is one of the most commonly performed orthopaedic operations. ${ }^{12} 13$ However, several studies have failed to show an advantage of arthroscopic surgery over conservative management or placebo surgery for these patients. ${ }^{13-15}$

There are few recent epidemiological and clinical studies on native joint infections (NJIs), which address the impact of iatrogenic infections. Two recent studies from New Zealand demonstrated that $16.9 \%$ and $17 \%$ of NJI were iatrogenic, respectively. ${ }^{47}$ A previous study in Iceland, 1990-2002, showed a rising incidence of SA, primarily due to 
cases related to open joint surgery and arthrocentesis. ${ }^{10}$ The proportion of iatrogenic infections was $41.8 \%$ among adults, making further studies on this problem imperative.

The primary objective of this retrospective, nationwide study was to describe the epidemiology, clinical characteristics and outcomes of NJI in Iceland during the subsequent 15 years, 2003-2017, and in particular, to assess the impact of iatrogenic infections in native joints.

\section{MATERIALS AND METHODS}

Setting, data sources and identification of positive cultures According to Statistics Iceland the country had 288471 inhabitants at the beginning of the study period and 348450 at the end. All inhabitants with residency of 6 months or more are covered by the Icelandic national health insurance, which is funded by taxes. Secondary care is provided by eight hospitals in the country, but vast majority of patients with joint infections are treated at two hospitals providing both secondary and tertiary care; most cultures of synovial fluid are performed in those two locations. Inpatient care is multidisciplinary, involving consultants in infectious disease, rheumatology and orthopaedics. A nationwide computerised and manual search for positive synovial fluid cultures was performed, covering a 15 -year period, 1 January 2003-31 December 2017, in all microbiology departments in Iceland. In cases where no antibiotic therapy was administered and/or symptoms were not compatible with infection, the isolate was considered a contaminant.

\section{Data collection, case definitions and exclusions}

Medical records of all patients with positive synovial fluid cultures taken from a native joint were reviewed for the following data: patient age, sex, comorbidities including presence of underlying joint disease, immunosuppressive treatment, joints involved, history of recent arthrocentesis, arthroscopy or joint surgery, clinical presentation, concomitant infection, laboratory test results, microbiology results, antimicrobial treatment and surgical management. Patients with contaminated cultures; those without compatible symptoms and/or those who did not receive any antimicrobial therapy were excluded. In a few cases of NJI, there was doubt whether the isolated pathogen was the real cause of infection or contamination. These cases were included if the patient had clinical signs of infection and received full antibiotic therapy for NJI.

Streptococcus pyogenes isolates were emm-typed and serotypes of Streptococcus pneumoniae were identified using standard methods.

Iatrogenic NJI was defined as infections diagnosed within 8 weeks following arthrocentesis or arthroscopy; or within 6 months from open joint surgery. Relapse of infection was defined as readmission with NJI of the previously infected joint within 6 months after completing treatment with reidentification of the offending organism.

Definitive treatment, with a microbiologically appropriate drug, was defined as therapy administered for more than $50 \%$ of the total duration of parenteral antimicrobial therapy.

\section{Epidemiology}

Overall, gender-specific and age-specific incidence rates were calculated by dividing the number of cases by the overall, genderspecific and age-specific Icelandic population as listed by Statistics Iceland and expressed as cases/100 000 individuals/year.

The total number of arthroscopic procedures performed during the study period was acquired from the Directorate of
Health in Iceland and used as the numerator in incidence calculations for arthroscopy-related infections as described above.

The prevalence of psoriatic arthritis (PsA) in Iceland is $0.14 \%{ }^{16}$ and the prevalence of rheumatoid arthritis (RA) is estimated between $0.8 \%$ and $1 \%$ based on previous Nordic studies. ${ }^{17}$ These numbers were used when calculating the incidence rates of $\mathrm{NJI}$ in patients with RA and PsA, presented as cases/1000 patients/year.

\section{Patient involvement}

There was no active patient or public involvement in this retrospective study.

\section{Statistical analysis}

Statistical analysis was performed using R V.3.1.3 (R Core Team, Vienna, Austria) and GraphPad Prism V.9.0 (GraphPad Software, San Diego, California, USA). Poisson regression analysis was performed for the age specific incidence of NJI. The $\chi^{2}$ test and Fisher's exact test were used for categorical variables and MannWhitney U test for continuous, non-normally distributed data. Multivariable logistic regression, adjusted for age and gender, was used with joint replacement surgery as the dependent variable and postarthroscopic NJI as the independent variable. Twotailed testing was performed and $p<0.05$ used as the level of significance.

\section{RESULTS}

\section{Identification of confirmed cases}

The process for identification of confirmed infections is shown in figure 1 . Over the 15 -year period, 893 microbiological samples from joint fluid were registered as positive. In 257 cases, the sample was incorrectly marked as joint fluid, most often coming from an infected bursa and 199 were contaminants. Therefore, culture-confirmed cases were identified in 437 patients, of which 299 were NJI.

\section{Demographics and clinical characteristics}

Overall, 40 children under the age of 18 and 259 adults fulfilled criteria for NJI, with a significant gender difference seen among adults (33\% women vs $67 \%$ men, $\mathrm{p}<0.001)$. The average age of adults was 60.2 years (SD 19) and children 6.4 years (SD 6.1) the youngest patient was 2 months old and the oldest 99 years old. The clinical and laboratory characteristics of NJI are shown by age in tables 1 and 2, respectively.

NJI occurred in nine patients with a history of injection drug use. In this patient group infection of the axial joints was significantly more common compared with non-injection drug users (4/9 vs 9/250, $\mathrm{p}<0.001)$.

A total of seven adult patients (2.7\%) were diagnosed with concurrent endocarditis and there was an increase during the study period with one case diagnosed in 2003-2010 and six cases in 2011-2017 (1/156 vs 6/143, p=0.05).

\section{Incidence}

The overall average incidence of culture confirmed NJI was 6.3/100 000 residents/year for the period 2003-2017, ranging from 3.7 to 10 cases $/ 100000$ residents (figure $2 \mathrm{~A}$ ). There was no significant change over the 15 -year time period. Age-specific and gender-specific incidence is shown in figure $2 \mathrm{~B}$, demonstrating the highest incidence rates at the extremes of the age spectrum, and consistent gender differences across almost all age groups. 




Figure 1 Flow chart showing identification of culture confirmed NJI in Iceland, 2003-2017. NJI, native joint infection.

\section{latrogenic infections}

NJI was considered iatrogenic in $7.5 \%$ of paediatric cases $(3 / 40)$ and $34 \%$ of adult cases (88/259). Infection was diagnosed following arthroscopy in 22.4\% (58/259) of cases, arthrocentesis in $7.3 \%(19 / 259)$ of cases and open joint surgery (other than joint replacement surgery) in $4.3 \%(11 / 259)$ of cases in adults (table 3). The median time from arthrocentesis to diagnosis of infection was 10 days (IQR 5.5-13 days) vs 13 days from arthroscopy (IQR 8-20.5 days) ( $\mathrm{p}=0.03)$. The median time from open joint surgery to diagnosis was 52 days (IQR 26.5-65 days). In adults $18-65$ years of age the overall percentage of iatrogenic infections was $45 \%$, significantly higher than the percentage of iatrogenic infections among older adults ( $\geq 65$ years) (67/149 vs $21 / 110, p<0.01)$. The difference was mostly due to postarthroscopic infections which were primarily observed in younger adults $(53 / 149$ vs $5 / 110, \mathrm{p}<0.01)$ whereas postarthrocentesis NJI was more common in older adults (7/149 vs $12 / 110$, $\mathrm{p}=0.09)($ table 1$)$.

When compared with a previous nationwide study covering 1990-2002, there was no significant change in the overall incidence of iatrogenic infections among adults (40/100 000/year in 1990-2002 vs 37.5/100 000/year in 2003-2017). There was a significant increase in infections among adults following arthroscopic procedures (9/100 000/year in 1990-2002 vs $25 / 100000 /$ year in 2003-2017, $\mathrm{p}<0.01)$. At the same time, the incidence of infections following arthrocentesis and open joint surgery (other than joint replacement surgery) decreased (17/100 000/year vs 8/100 000/year for arthrocentesis, $p=0.1$; $13.5 / 100000 /$ year vs 5/100 000/year, $\mathrm{p}=0.06$ for open joint surgery, respectively).
During the whole study period at least 22033 arthroscopic procedures were performed. However, due to incomplete data before 2010 the number of iatrogenic infections following arthroscopic procedures during 2010-2017 was used to calculate the risk per procedure. The estimated ratio of infections per arthroscopic procedure is shown in table 4 . The knee joint was analysed separately since the majority of iatrogenic infections followed procedures on the knee.

\section{Contributing risk factors}

Overall, $82 \%$ of adults had a potential risk factor for NJI (online supplemental table 1). Underlying joint disease was present in $49 \%(127 / 259)$, with osteoarthritis being most common. Inflammatory rheumatic disease was present in $24 \%$ with crystal arthropathy being most common. There was a decrease in the incidence of infections in patients with RA and PsA during the study period (6.7 cases/1000 patients/year in 20032010 vs 2 cases/1000 patients/year in 2011-2017, p $=0.04$ and 15.9 cases $/ 1000$ patients/year vs 5.8 cases $/ 1000$ patients/year, $\mathrm{p}=0.3$, respectively).

\section{Microbial aetiology}

Bacterial species isolated from joint samples are shown in table 5. Methicillin-susceptible-Staphylococcus aureus was most common in children and adults, followed by different streptococcal species in adults. Coagulase-negative staphylococci were significantly more common in NJI following any kind of joint procedure $(33 / 88$ vs $10 / 171, \mathrm{p}<0.01)$. Kingella kingae was the second most common isolate in children and was only found in 
Table 1 Clinical characteristics on admission among children and adults with NJI in Iceland, 2003-2017

\begin{tabular}{|c|c|c|c|c|c|c|c|}
\hline & Children $<2$ years & $2-18$ years & Total & Adults $18-65$ years & $>65$ years & Total & $P$ value \\
\hline No of cases & 19 & 21 & 40 & 149 & 110 & 259 & \\
\hline Time to presentation (days) & 2 & 3 & 3 & 4 & 2 & 3 & \\
\hline Joint pain & $16 / 19(84)$ & $21 / 21(100)$ & $37 / 40(93)$ & $146 / 149(98)$ & $108 / 110(98)$ & $254 / 259$ (98) & 0.08 \\
\hline Swollen joint* & $14 / 18(78)$ & $20 / 21(95)$ & $31 / 39(79)$ & $144 / 148(97)$ & $101 / 107(94)$ & $245 / 255$ (96) & $<0.001$ \\
\hline DROMT & $19 / 19(100)$ & $20 / 20(100)$ & $39 / 39(100)$ & 141/142 (99) & $104 / 107(97)$ & $245 / 249$ (98) & 1 \\
\hline Joint redness & $8 / 15(53)$ & $7 / 13(54)$ & $15 / 28(54)$ & $43 / 112(38)$ & $47 / 90(52)$ & $90 / 202(45)$ & 0.4 \\
\hline Warm joint $\ddagger$ & $8 / 12(67)$ & $11 / 13(85)$ & $19 / 25(76)$ & $89 / 115(77)$ & $54 / 72(75)$ & $143 / 187(76.5)$ & 1 \\
\hline \multicolumn{8}{|l|}{ Infected joint: } \\
\hline Knee & $4 / 19(21)$ & $9 / 21(43)$ & $13 / 40(33)$ & $95 / 149(64)$ & $53 / 110(48)$ & $148 / 259(57)$ & 0.006 \\
\hline Shoulder & $3 / 19(16)$ & 0 & $3 / 40(7.5)$ & $7 / 149(5)$ & $18 / 110(16)$ & $25 / 259(10)$ & 1 \\
\hline Elbow & $2 / 19(11)$ & $7 / 21(33)$ & $9 / 40(23)$ & $1 / 149(1)$ & 0 & $1 / 259(0.5)$ & $<0.001$ \\
\hline Hip & $1 / 19(5)$ & $3 / 21(14)$ & $4 / 40(10)$ & $12 / 149(8)$ & $8 / 110(7)$ & $20 / 259(8)$ & 0.5 \\
\hline Ankle & $6 / 19(32)$ & $2 / 21(9.5)$ & $8 / 40(20)$ & $11 / 149(7)$ & $7 / 110(6)$ & $18 / 259(7)$ & 0.01 \\
\hline Wrist & $1 / 19(5)$ & 0 & $1 / 40(2.5)$ & $3 / 149(2)$ & $14 / 110(13)$ & $17 / 259(6.5)$ & 0.5 \\
\hline Hands and feet & 0 & 0 & 0 & $5 / 149(3)$ & $6 / 110(5)$ & $11 / 259$ (4) & - \\
\hline Other joints§ & $2 / 19(11)$ & 0 & $2 / 40(5)$ & $15 / 149(10)$ & $4 / 110(4)$ & $19 / 259(7)$ & 1 \\
\hline Polyarticular infection & 0 & 0 & 0 & $7 / 149(5)$ & $12 / 110(11)$ & $19 / 259(7)$ & - \\
\hline latrogenic infection & 0 & $3 / 21(14)$ & $3 / 40(7.5)$ & $67 / 149(45)$ & $21 / 110(19)$ & $88 / 259(34)$ & $<0.001$ \\
\hline
\end{tabular}

Data are no/no (\%).

$P$ value, children vs adults.

$P$ values $<0.05$ are shown in bold.

*Information on joint swelling found in: $18 / 19$ cases $<2$ years, $21 / 21$ cases $2-18$ years, 148/149 cases $18-65$ years and $107 / 110>65$ years.

tDROM: Information found in: $19 / 19$ cases <2 years, 20/21 cases 2-18 years, 142/149 cases 18-65 years and $107 / 110>65$ years.

¥Information on temperature change over joint found in: $12 / 19$ cases $<2$ years, $13 / 21$ cases $2-18$ years, $115 / 149$ cases $18-65$ years and $72 / 110>65$ years.

§Adults: 8 sternoclavicular, 7 acromioclavicular, 2 sacroiliac, 2 vertebral facet joint infections. Children: 2 sacroiliac infections.

IInformation on joint redness found in: $15 / 19$ cases $<2$ years, 13/21 cases $2-18$ years, 112/149 cases $18-65$ years and 90/110 >65 years.

DROM, decreased range of motion; NJI, native joint infection.

children $<3$ years of age. $S$. aureus was the causative pathogen in $78 \%$ of infections among injection drug users and $56 \%$ of patients with RA. There was an increase in the number of NJI caused by $S$. pyogenes during the study period, with two infections diagnosed in 2003-2010 and 8 in 2011-2017 (2/156 vs $8 / 143, \mathrm{p}=0.05)$.

\section{Treatment and outcomes}

Empiric and definitive antimicrobial therapy are summarised in online supplemental table 2, treatment and outcomes are summarised in online supplemental table 3. Median duration of parenteral antimicrobial treatment was significantly longer in adults compared with children, 29 days in adults (IQR $=21-42$ days) vs 13.5 days in children (IQR $=10-28.5$ days) $(\mathrm{p}<0.01)$. In adults, 24 patients (9\%) received a prosthetic joint following infection. The average time from diagnosis to arthroplastic surgery was 3.5 years. Of these patients, 62.5\% (15/24) had a history of iatrogenic infection with $46 \%$ (11/24) having postarthroscopic infection (11/58 vs $13 / 201, \mathrm{p}=0.008)$ and $16.7 \%(4 / 24)$ having infection following arthrocentesis (4/19 vs $20 / 240, p=0.08)$. Postarthroscopic NJI was independently associated with the need for subsequent arthroplastic surgery after adjusting for age and gender with OR 3.6 (95\% CI 1.3 to 10.3). The average time from postarthroscopic NJI to insertion of a prosthetic joint was 3.2 years.

Relapse of infection after treatment occurred in $4.6 \%$ of adult cases $(12 / 259)$ with no relapse noted in paediatric cases of NJI. The average age of these patients was 61 years and 50\% (6/12) had an iatrogenic infection (postarthroscopy four patients, postarthrocentesis one patient, open joint surgery one patient; $\mathrm{p}=0.2)$. S. aureus was the pathogen in $75 \%(9 / 12)$ and the median time of parenteral therapy before relapse was 29 days (IQR=21-41).

The overall 30-day mortality rate was 4.7\% (14/299) and in adults the mortality was $5.4 \%(14 / 259)$. The average age of these patients was 81 years. Of those who died $50 \%$ had $S$. aureus infection. Deaths could be directly attributed to the infection in $57 \%$ of cases $(8 / 14)$ while it was contributory in $43 \%$.

\section{DISCUSSION}

This comprehensive nationwide study provides epidemiological, clinical and prognostic analysis of patients with cultureproven NJI over a 15 -year period. The average incidence of NJI in Iceland 2003-2017 was 6.3 cases/100 000 residents per year, with the highest incidence rates seen at the extremes of the age spectrum, as found in previous studies. ${ }^{10} 1118$ The male predominance observed among NJI is also in line with earlier reports. ${ }^{10111920}$

The median time from the onset of symptoms to healthcare presentation and subsequent diagnosis in our study was 3 days, a markedly shorter time than previously reported. ${ }^{12} 21$ One explanation for this shorter time may be universal ease of access to healthcare services in Iceland.

The proportion of patients with iatrogenic infection was $34 \%$ in adults, considerably higher than has been reported by others. ${ }^{47}$ In young adults (18-65 years), nearly half of the infections were iatrogenic, which is alarmingly high. Previously, the mean number of iatrogenic infections rose from 2.8 annual infections in 1990-1994 to 9 infections/year in 1998-2002, ${ }^{10}$ but according to our results they have since remained stable. The infections most often followed arthroscopic procedures while in the previous decade infection following arthrocentesis was most 
Table 2 Investigations and laboratory results on diagnosis among children and adults with NJI in Iceland, 2003-2017

\begin{tabular}{|c|c|c|c|c|c|c|c|}
\hline & Children $<2$ years & $2-18$ years & Total & Adults $18-65$ years & $>65$ years & Total & $P$ value \\
\hline No of cases & 19 & 21 & 40 & 149 & 110 & 259 & \\
\hline \multicolumn{8}{|l|}{ Admission data: } \\
\hline Temperature (median, ${ }^{\circ} \mathrm{C}$ ) & 38 & 38 & 38 & 37.7 & 37.6 & 37.7 & 0.02 \\
\hline WCC (median, $\times 10^{9} / \mathrm{L}$ ) & 13.2 & 10.9 & 11.4 & 9.9 & 11.6 & 10.5 & 0.4 \\
\hline ESR (median, mm/hour) & 34 & 35 & 35 & 54 & 71 & 57 & 0.006 \\
\hline CRP (median, mg/L) & 49 & 48 & 49 & 123 & 180 & 148 & $<0.001$ \\
\hline Synovial fluid WCC (median, $\left.\times 10^{6}\right)^{*}$ & 86675 & 31075 & 31075 & 50400 & 69000 & 58794 & 0.3 \\
\hline Positive blood culturet & $3 / 16(19)$ & $7 / 15(47)$ & $10 / 31(32)$ & $32 / 100(32)$ & $42 / 79(53)$ & $74 / 179(41)$ & 0.4 \\
\hline Crystal arthropathy & - & - & - & $5 / 60(8)$ & $22 / 63(35)$ & $27 / 123(22)$ & - \\
\hline \multicolumn{8}{|l|}{ Normal admission values: $\ddagger$} \\
\hline Temperature $<37.8^{\circ} \mathrm{C}$ & $6 / 19(32)$ & $8 / 21(38)$ & $14 / 40(35)$ & $78 / 141(55)$ & $59 / 102(58)$ & $137 / 246(56)$ & 0.02 \\
\hline WCC $<10.5 \times 10^{9} / \mathrm{L}$ & $6 / 19(32)$ & $10 / 21(48)$ & $16 / 40(40)$ & $84 / 143(59)$ & $41 / 109(38)$ & $125 / 252(50)$ & 0.3 \\
\hline CRP $<10 \mathrm{mg} / \mathrm{L}$ & $2 / 19(10.5)$ & 4/21 (19) & $6 / 40(15)$ & $4 / 143(3)$ & $6 / 107(6)$ & $10 / 250(4)$ & 0.01 \\
\hline ESR $<20 \mathrm{~mm} /$ hour & $1 / 10(10)$ & $1 / 7(14)$ & $2 / 17(12)$ & $11 / 70(16)$ & $7 / 49(14)$ & $18 / 119(15)$ & 1 \\
\hline \multicolumn{8}{|l|}{ Other infective syndromes: } \\
\hline Adjacent osteomyelitis & $3 / 19(16)$ & 9/21 (43) & $12 / 40(30)$ & $16 / 149(11)$ & $13 / 110(12)$ & $29 / 259(11)$ & 0.005 \\
\hline Endocarditis & 0 & 0 & 0 & $3 / 149(2)$ & $4 / 110(4)$ & $7 / 259(3)$ & - \\
\hline Cellulitis & 0 & 0 & 0 & $3 / 149(2)$ & $3 / 110(3)$ & $6 / 259(2)$ & - \\
\hline Other§ & 0 & 0 & 0 & $3 / 149(2)$ & $5 / 110$ (5) & $8 / 259$ (3) & - \\
\hline
\end{tabular}

Data are no/no (\%).

$P$ value, children vs adults.

$P$ values $<0.05$ are shown in bold.

*Information on number of WCC in the synovial fluid obtained was found in 171 adult cases and 12 paediatric cases.

†Information on blood culture (whether taken, positive or negative) was found in: all paediatric cases, 141 cases in age group 18-65 years and 103 cases $>65$ years. Blood culture was performed in $16 / 19<2$ years, $15 / 21$ cases $2-18$ years, $100 / 149$ cases $18-65$ years and 79/110 $>65$ years.

fInformation on (A) temperature was found in: all paediatric cases, 144/149 cases 18-65 years and 102/110 >65 years. (B) WCC: all paediatric cases, 143/149 cases 18-65 years and $109 / 110>65$ years. (C) CRP: all paediatric cases, $143 / 149$ cases $18-65$ years, 107/110>65 years. (D) ESR: $10 / 19$ cases <2 years, 7/21 cases $2-18$ years, 70/149 cases $18-65$ years and $49 / 110>65$ years.

§Pneumonia 5 (all patients were $>65$ years), urinary tract infection 2, streptococcal toxic shock syndrome one patient.

IInformation on concurrent crystal arthropathy in adults was found in: 60/149 cases 18-65 and 63/110>65 years.

CRP, C reactive protein; ESR, erythrocyte sedimentation rate; NJl, native joint infection; WCC, white cell count.

common (online supplemental figure 2). The frequency of postarthroscopic NJI in older reports ranges from $0.1 \%$ to $0.5 \%{ }^{10} 22$ with more recent publications reporting $0.15 \%-0.84 \% .^{23-25}$ Our study estimated the frequency of postarthroscopic NJI to be $0.17 \%$, with $0.24 \%$ for the knee joint, which is consistent with these reports.

Although the frequency of postarthroscopic arthritis per procedure has remained stable over the last 15 years, the number of infections has increased due to an increase in the number of arthroscopic procedures. The Directorate of Health in Iceland published a report in 2017 on the yearly numbers of arthroscopic knee procedures performed in Iceland. From 2012 to 2016, the frequency was estimated to be 590 arthroscopies/100 000 residents/year and 890 procedures/100 000 residents over the age of $50 .^{26}$ These numbers are considerably higher than those published from other Nordic countries. In 2011, the frequency of arthroscopies among patients older than 55 years in Denmark was 322 procedures/100 000 residents and in 2012 the frequency of knee arthroscopies in patients older than 18 years in Finland was $347 / 100000 .^{27} 28$ For further comparison the frequency in Iceland in 2012 in patients older than 18 years was 770 knee arthroscopies/100 000 residents. ${ }^{26}$ This indicates that arthroscopic knee procedures are overused in Iceland and since the evidence of their benefit is weak with previous studies consistently showing that APM, one of the most commonly performed arthroscopic procedure, offers no benefit over conservative treatment or placebo surgery, the indications for these procedures should be reviewed. ${ }^{12-15}$ Additionally, other potential complications including pulmonary embolism, cast this wide practice in doubt. ${ }^{29}$

A high number of adult NJI patients had an underlying joint disease $(49 \%)$ of which $24 \%$ had inflammatory rheumatic disease and just over $6 \%$ of patients had RA, which is considered to be a major risk factor for NJI. This proportion of patients with RA was lower than reported in some studies, ${ }^{121}$ yet similar to some. ${ }^{3} 418$ These data, therefore, suggest that it may be inflammatory joint disease itself rather than the specific disease process which raises risk of NJI. A decrease was seen in the incidence of NJI in patients with RA and PsA over the study period. Although immunosuppressive drugs such as disease-modifying antirheumatic drug and TNF-alpha inhibitors are considered to increase the risk of infections, it is possible that their role in preserving function and subsequent decreased need for arthrocentesis and intra-articular steroids may offer overall protection against infection.

The median duration of parenteral antimicrobial treatment for NJI was significantly longer for adults (4 weeks) compared with children (2 weeks) (online supplemental table 3 ). The optimal duration of antimicrobial therapy in children has been studied with prospective, randomised trials showing that treatment with intravenous antibiotics for short periods (4-7 days), followed by oral therapy was as successful as longer courses of parenteral therapy $^{30} 31$ and guidelines for treatment were changed accordingly in 2011. There is less consensus on the duration of treatment for NJI in adults. The general recommendation is 2-4 weeks of intravenous treatment followed by oral therapy for at 
Incidence of NJI in Iceland 2003-2017

12

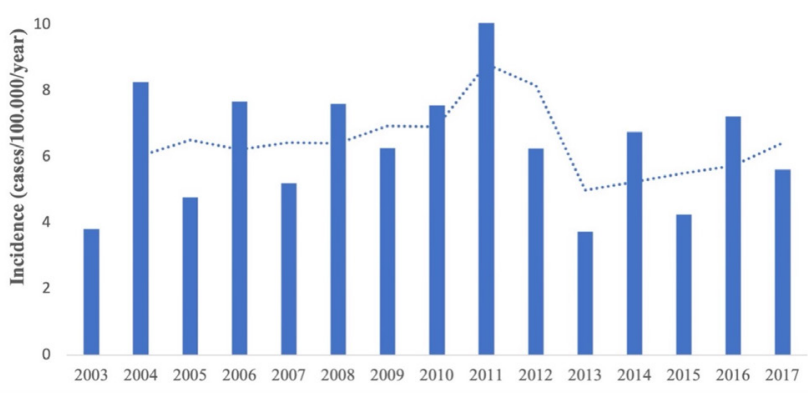

$40 \quad$ Age and gender-specific incidence

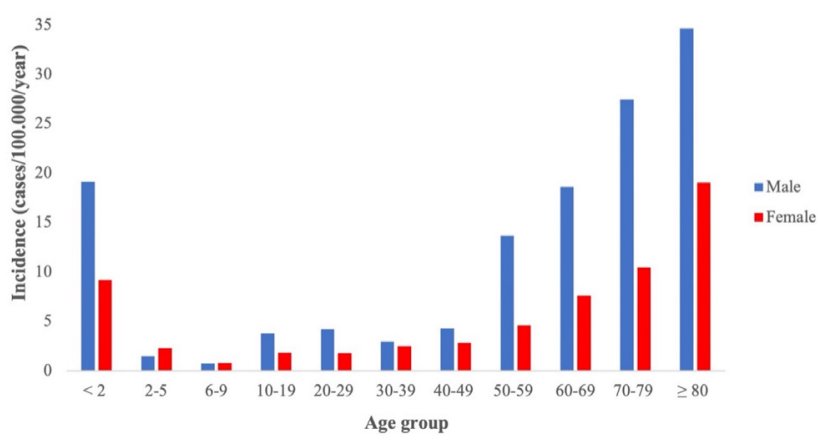

Figure 2 (A) Overall annual incidence of culture confirmed NJI in Iceland, 2003-2017. The dashed line shows running 2-year average. (B) Age-specific and gender-specific incidence of culture confirmed NJI in Iceland, 2003-2017. NJI, native joint infection.

least 7-14 days. This is consistent with the median duration of parenteral antibiotic therapy in adults in our study, but it is probable that the time could have been shortened in selected cases, as suggested by more recent studies. ${ }^{732}$

The mortality rate in our study was $5.4 \%$ in adults, which is in the lower range compared with previous reports. ${ }^{124-68}$ We found that $9 \%$ of adults in our cohort required arthroplastic surgery, all within 9 years of diagnoses of NJI. A large retrospective cohort
Table 4 Ratio of infections per arthroscopic procedure, 2010-2017

\begin{tabular}{llll}
\hline Joint & $\begin{array}{l}\text { No of SA following } \\
\text { arthroscopy }\end{array}$ & $\begin{array}{l}\text { No of arthroscopic } \\
\text { procedures }\end{array}$ & Ratio, \% \\
\hline All joints & 37 & 21342 & 0.17 \\
Knee joint & 32 & 13290 & 0.24 \\
\hline Other joints & 5 & 8052 & 0.06 \\
\hline
\end{tabular}

*Shoulder joint: 4, ankle joint: 1 .

SA, septic arthritis.

study on patients who received arthroscopic knee washout for NJI between 1997 and 2017 in England, found that within 15 years, $9 \%$ of patients had knee arthroplasty, corresponding to an annual rate of arthroplasty about six times higher than in the general population. ${ }^{8}$ These findings highlight the potential risk of long-term consequences following NJI. Our study found that patients with a history of postarthroscopic infection are significantly more likely to require arthroplastic surgery compared with other patients with NJI, after adjusting for age and gender. Previous cohort studies suggest that progression of osteoarthritis may be more rapid in those who have undergone arthroscopic procedures, possibly accelerating the need for arthroplastic surgery. ${ }^{33} 34$ When bacterial infection complicates these procedures, more rapid destruction of the joint is likely to further accelerate the need for joint replacement.

The comprehensive nationwide approach of this study as well as the microbiological confirmation of cases is the main strengths of this study. Moreover, detailed clinical and follow-up information was available for most cases and historical information was available for epidemiological comparisons. Nevertheless, this study has important limitations. The number of NJI in our study is underestimated due to limitation of case ascertainment to culture positivity. Case ascertainment using diagnostic codes or Newmans's modified criteria would likely have increased our case numbers. In particular, cases of small joint NJI are underrepresented in our cohort since synovial fluid sampling is often not performed when small joint NJI is suspected as these joints often do not have enough volume to support needle aspiration. ${ }^{7}$ Similarly, it is likely that the incidence of NJI in young children is underestimated in our study as cultures of synovial fluid are the mainstay of diagnosis while PCR for K. kingae has been shown to

Table 3 latrogenic NJI in adults in Iceland 2003-2017

\begin{tabular}{|c|c|c|c|c|}
\hline & Arthrocentesis & Arthroscopy & Open joint surgery & Total \\
\hline No of cases & 19 & 58 & 11 & 88 \\
\hline Age (years, median) & 69 & 54 & 63 & 57 \\
\hline Time from procedure to diagnosis (days, median) & 10 & 13 & 52 & 13 \\
\hline Male/female ratio & 1.4 & 2.6 & 2.7 & 2.3 \\
\hline \multicolumn{5}{|l|}{ Infected joint } \\
\hline Knee & $11 / 19(58)$ & $52 / 58(90)$ & 8/11 (73) & $71 / 88(81)$ \\
\hline Shoulder & $3 / 19(16)$ & $4 / 58(7)$ & $1 / 11(9)$ & $8 / 88(9)$ \\
\hline Other* & $5 / 19(26)$ & $2 / 58(3)$ & 2/11 (18) & $9 / 88(10)$ \\
\hline \multicolumn{5}{|l|}{ Synovial culture } \\
\hline Staphylococcus aureus & $11 / 19(58)$ & $23 / 58(40)$ & $4 / 11(36)$ & $38 / 88(43)$ \\
\hline CNS & $4 / 19(21)$ & $23 / 58(40)$ & $6 / 11(55)$ & $33 / 88(38)$ \\
\hline Streptococcit & $3 / 19(16)$ & $5 / 58(9)$ & $1 / 11(9)$ & $9 / 88(10)$ \\
\hline Otherł‡ & $1 / 19(5)$ & $7 / 58(12)$ & 0 & $8 / 88(9)$ \\
\hline
\end{tabular}

Data are no/no (\%). Median values for age and time from procedure to diagnosis are shown.

${ }^{*}$ Acromioclavicular, ankle, hip, proximal interphalangeal and wrist joint.

†Streptococcus, viridans group: 3, Streptococci group C or G: 4, Streptococcus mitis: 1, Streptococcus pneumoniae: 1.

$\ddagger$ Enterobacter cloacae, gram positive rods, Klebsiella oxytoca, Lactococcus cremoris, Micrococcus species.

CNS, coagulase-negative staphylococci; NJI, native joint infection. 


\begin{tabular}{|c|c|c|c|}
\hline Bacterial isolates & $\begin{array}{l}\text { Children }(<18 \\
\text { years) } n=40\end{array}$ & $\begin{array}{l}\text { Adults }(\geq 18 \\
\text { years) } n=259\end{array}$ & $\begin{array}{l}\text { Total (\%) } \\
\mathrm{n}=299\end{array}$ \\
\hline Staphylococcus aureust† & $19(48)$ & $137(53)$ & $156(52)$ \\
\hline Staphylococcus lugdunensis & 0 & $7(3)$ & $7(2)$ \\
\hline $\begin{array}{l}\text { Coagulase-negative } \\
\text { Staphylococci }\end{array}$ & $1(2.5)$ & $43(17)$ & $44(15)$ \\
\hline Streptococcus agalactiae & 0 & $8(3)$ & $8(3)$ \\
\hline Streptococcus pneumoniae* & $1(2.5)$ & $8(3)$ & $9(3)$ \\
\hline Streptococcus pyogenes $\dagger$ & $1(2.5)$ & $9(3.5)$ & $10(3)$ \\
\hline Streptococci group C or G & $1(2.5)$ & $20(8)$ & $21(7)$ \\
\hline Other Streptococci & $2(5)$ & $8(3)$ & $10(3)$ \\
\hline All Streptococci & $5(12.5)$ & $53(20)$ & $58(19)$ \\
\hline Escherichia coli & 0 & $3(1)$ & $3(1)$ \\
\hline Enterobacter cloacae & 0 & $4(1.5)$ & $4(1)$ \\
\hline Kingella kingae & $11(28)$ & 0 & $11(4)$ \\
\hline Other gram-negative rods $\ddagger$ & $1(2.5)$ & $6(2)$ & $7(2)$ \\
\hline All gram-negative rods & $12(30)$ & $13(5)$ & $25(8)$ \\
\hline Neisseria gonorrhoea & 0 & $1(0.4)$ & $1(0.5)$ \\
\hline Gram positive rods§ & $3(7.5)$ & $4(1.5)$ & $7(2)$ \\
\hline Otherף & $1(2.5)$ & $6(2)$ & $7(2)$ \\
\hline Total $^{* *}$ & 41 & 264 & 305 \\
\hline
\end{tabular}

Data are no/no (\%).

*Serotypes in year 2003: 22, 2004: 4, 12, 35, 2007: 19F, 6B, 2009: 6B, 2014: 21, 2016: 15B/C

tInformation on emm types was available in 9/10 isolates. Overall, seven different emm types were identified, the most common type was emm3 (33\%), all three cases isolated after 2013. Other types were emm1, 12, 28, 81, 87, 89.

$\ddagger$ Burkholderia pseudomallei, Escherichia hermannii, Klebsiella oxytoca, Pasteurella multocida, Pseudomonas aeruginosa, Rosemonas species, Stenotrophomonas maltophilia.

$\S B$ acillus species, Clostridium perfringens, Corynebacterium species, gram positive rods unspecified.

IAerococcus urinae, Enterococcus faecium, Lactococcus cremoris, Micrococcus species, anaerobic gram-positive cocci unspecified.

${ }^{* *}$ Four patients had two bacterial species isolated, one had three.

t†Only Methicillin-susceptible $S$. aureus was isolated.

$\mathrm{NJI}$, native joint infection.

have significantly better sensitivity. It is, however, unlikely that this will influence the rate of complications as the clinical course of $K$. kingae NJI is usually benign. ${ }^{35} 36$

\section{CONCLUSION}

This study suggests that the incidence of NJI in Iceland has remained stable over the past 15 years. However, the proportion of iatrogenic infections is high, seen in $45 \%$ of young adults, most often following arthroscopic procedures which are increasingly being performed on relatively young patients with joint complaints. Although the overall frequency of postarthroscopic infections remains unchanged, the incidence of these infections has significantly increased when compared with the previous decade due to more widespread use. Our study suggests that patients with postarthroscopic infection are more likely to relapse after treatment when compared with other patients with NJI and are more likely to receive arthroplastic surgery. Despite being rare, there are serious complications associated with arthroscopy, including infection which entails considerable morbidity. Appreciation of these risks and establishment of firm indications for arthroscopic procedures is essential.

Acknowledgements We thank Bergdis Bjork Sigurjonsdottir, project manager at Directorate of health in Iceland for assistance in data gathering and Kristjan Godsk Rognvaldsson for review and assistance with statistical analysis.
Contributors MG had full access to all of the data in the study and takes responsibility for the integrity of the data and the accuracy of the data analysis. Study conception and design: SLG, HE, SG and MG. Acquisition of data: SLG, HE and MG. Analysis and interpretation of data: SLG and MG. All authors were involved in drafting the article or revising it critically for important intellectual content, and all authors approved the final version to be published.

Funding This study was supported by a grant from the Science Fund, Landspitali University Hospital.

Competing interests None declared.

\section{Patient consent for publication Not required.}

Ethics approval This study was approved by the National Bioethics Committee. Reference number 15-008-V2. The requirement for informed consent was waived as this was a retrospective study with no direct participation by patients.

Provenance and peer review Not commissioned; externally peer reviewed.

Data availability statement All data relevant to the study are included in the article or uploaded as online supplemental information.

Supplemental material This content has been supplied by the author(s). It has not been vetted by BMJ Publishing Group Limited (BMJ) and may not have been peer-reviewed. Any opinions or recommendations discussed are solely those of the author(s) and are not endorsed by BMJ. BMJ disclaims all liability and responsibility arising from any reliance placed on the content. Where the content includes any translated material, BMJ does not warrant the accuracy and reliability of the translations (including but not limited to local regulations, clinical guidelines, terminology, drug names and drug dosages), and is not responsible for any error and/or omissions arising from translation and adaptation or otherwise.

Open access This is an open access article distributed in accordance with the Creative Commons Attribution Non Commercial (CC BY-NC 4.0) license, which permits others to distribute, remix, adapt, build upon this work non-commercially, and license their derivative works on different terms, provided the original work is properly cited, appropriate credit is given, any changes made indicated, and the use is non-commercial. See: http://creativecommons.org/licenses/by-nc/4.0/.

\section{ORCID iD}

Magnús Gottfreðsson http://orcid.org/0000-0003-2465-0422

\section{REFERENCES}

1 Gupta MN, Sturrock RD, Field M. A prospective 2-year study of 75 patients with adultonset septic arthritis. Rheumatology 2001;40:24-30.

2 Nolla JM, Lora-Tamayo J, Gómez Vaquero C, et al. Pyogenic arthritis of native joints in non-intravenous drug users: a detailed analysis of 268 cases attended in a tertiary hospital over a 22-year period. Semin Arthritis Rheum 2015;45:94-102.

3 Ross JJ, Ard KL, Carlile N. Septic arthritis and the opioid epidemic: 1465 cases of culture-positive native joint septic arthritis from 1990-2018. Open Forum Infect Dis 2020;7:ofaa089.

4 Kennedy N, Chambers ST, Nolan I, et al. Native joint septic arthritis: epidemiology, clinical features, and microbiological causes in a new Zealand population. J Rheumatol 2015;42:2392-7

5 Huang Y-C, Ho C-H, Lin Y-J, et al. Site-Specific mortality in native joint septic arthritis: a national population study. Rheumatology 2020;59:3826-33.

6 Andreasen RA, Andersen NS, Just SA, et al. Prognostic factors associated with mortality in patients with septic arthritis: a descriptive cohort study. Scand J Rheumatol 2017;46:27-32.

7 McBride S, Mowbray J, Caughey W, et al. Epidemiology, management, and outcomes of large and small native joint septic arthritis in adults. Clin Infect Dis 2020;70:271-9.

8 Abram SGF, Alvand A, Judge A, et al. Mortality and adverse joint outcomes following septic arthritis of the native knee: a longitudinal cohort study of patients receiving arthroscopic washout. Lancet Infect Dis 2020;20:341-9.

9 Weston VC, Jones AC, Bradbury N, et al. Clinical features and outcome of septic arthritis in a single UK health district 1982-1991. Ann Rheum Dis 1999;58:214-9.

10 Geirsson AJ, Statkevicius S, Víkingsson A. Septic arthritis in Iceland 1990-2002: increasing incidence due to iatrogenic infections. Ann Rheum Dis 2008;67:638-43.

11 Rutherford Al, Subesinghe S, Bharucha T, et al. A population study of the reported incidence of native joint septic arthritis in the United Kingdom between 1998 and 2013. Rheumatology 2016;55:2176-80.

12 Kim S, Bosque J, Meehan JP, et al. Increase in outpatient knee arthroscopy in the United States: a comparison of national surveys of ambulatory surgery, 1996 and 2006. J Bone Joint Surg Am 2011;93:994-1000.

13 Sihvonen R, Paavola M, Malmivaara A, et al. Arthroscopic partial meniscectomy versus placebo surgery for a degenerative meniscus tear: a 2-year follow-up of the randomised controlled trial. Ann Rheum Dis 2018;77:188-95.

14 Khan M, Evaniew N, Bedi A, et al. Arthroscopic surgery for degenerative tears of the meniscus: a systematic review and meta-analysis. CMAJ 2014;186:1057-64.

15 Sihvonen R, Paavola M, Malmivaara A, et al. Arthroscopic partial meniscectomy versus sham surgery for a degenerative meniscal tear. N Engl J Med 2013;369:2515-24. 
16 Love TJ, Gudbjornsson B, Gudjonsson JE, et al. Psoriatic arthritis in Reykjavik, Iceland: prevalence, demographics, and disease course. J Rheumatol 2007;34:2082-8.

17 Neovius M, Simard JF, Askling J, et al. Nationwide prevalence of rheumatoid arthritis and penetration of disease-modifying drugs in Sweden. Ann Rheum Dis 2011;70:624-9.

18 Kaandorp CJ, Dinant HJ, van de Laar MA, et al. Incidence and sources of native and prosthetic joint infection: a community based prospective survey. Ann Rheum Dis 1997:56:470-5

19 Vassallo C, Borg AA, Farrugia D, et al. The epidemiology and outcomes of septic arthritis in the Maltese Islands: a hospital-based retrospective cohort study. Mediterr J Rheumatol 2020;31:195-205.

20 Nissim L, Lieber SB, Naffaa ME, et al. The impact of gender on the clinical presentation, management, and surgical outcomes of patients with native-joint septic arthritis. J Eval Clin Pract 2021;27:371-6.

21 Favero M, Schiavon F, Riato $L$, et al. Rheumatoid arthritis is the major risk factor for septic arthritis in rheumatological settings. Autoimmun Rev 2008;8:59-61.

22 Kuzmanova SI, Atanassov AN, Andreev SA, et al. Minor and major complications of arthroscopic synovectomy of the knee joint performed by rheumatologist. Folia Med 2003:45:55-9.

23 Yeranosian MG, Petrigliano FA, Terrell RD, et al. Incidence of postoperative infections requiring reoperation after arthroscopic knee surgery. Arthroscopy 2013;29:1355-61.

24 Salzler MJ, Lin A, Miller CD, et al. Complications after arthroscopic knee surgery. Am J Sports Med 2014;42:292-6.

25 Clement RC, Haddix KP, Creighton RA, et al. Risk factors for infection after knee arthroscopy: analysis of 595,083 cases from 3 United States databases. Arthroscopy 2016:32:2556-61.

26 Jakobsson B, Gudlaugsson J, Haraldsdottir S. Tiðni aðgerða i einkarekinni pjónustu 2007-2016. Reykjavik: Directorate Of Health in Iceland, 2017: 11.

27 Mattila VM, Sihvonen R, Paloneva J, et al. Changes in rates of arthroscopy due to degenerative knee disease and traumatic meniscal tears in Finland and Sweden. Acta Orthop 2016:87:5-11.
28 Thorlund JB, Hare KB, Lohmander LS. Large increase in arthroscopic meniscus surgery in the middle-aged and older population in Denmark from 2000 to 2011. Acta Orthop 2014;85:287-92

29 Abram SGF, Judge A, Beard DJ, et al. Adverse outcomes after arthroscopic partial meniscectomy: a study of 700000 procedures in the national Hospital Episode Statistics database for England. Lancet 2018;392:2194-202.

30 Peltola $\mathrm{H}$, Pääkkönen $\mathrm{M}$, Kallio $\mathrm{P}$, et al. Prospective, randomized trial of 10 days versus 30 days of antimicrobial treatment, including a short-term course of parenteral therapy, for childhood septic arthritis. Clin Infect Dis 2009:48:1201-10.

31 Jaberi FM, Shahcheraghi GH, Ahadzadeh M. Short-term intravenous antibiotic treatment of acute hematogenous bone and joint infection in children: a prospective randomized trial. J Pediatr Orthop 2002;22:317-20.

32 Gjika E, Beaulieu J-Y, Vakalopoulos K, et al. Two weeks versus four weeks of antibiotic therapy after surgical drainage for native joint bacterial arthritis: a prospective, randomised, non-inferiority trial. Ann Rheum Dis 2019;78:1114-21.

33 Roos EM, Ostenberg A, Roos H, et al. Long-term outcome of meniscectomy: symptoms, function, and performance tests in patients with or without radiographic osteoarthritis compared to matched controls. Osteoarthritis Cartilage 2001;9:316-24.

34 Englund M, Lohmander LS. Risk factors for symptomatic knee osteoarthritis fifteen to twenty-two years after meniscectomy. Arthritis Rheum 2004;50:2811-9.

35 Hernández-Rupérez MB, Suárez-Arrabal MDC, Villa-García Ángel, et al. Kingella kingae as the main cause of septic arthritis: importance of molecular diagnosis. Pediatr Infect Dis J 2018;37:1211-6.

36 Gouveia C, Duarte M, Norte $\mathrm{S}$, et al. Kingella kingae displaced S. aureus as the most common cause of acute septic arthritis in children of all ages. Pediatr Infect Dis $J$ 2021;40:623-7. 